\title{
Article \\ Experimental Study of Plasma Plume Analysis of Long Pulse Laser Irradiates CFRP and GFRP Composite Materials
}

\author{
Yao Ma, Chao Xin, Wei Zhang and Guangyong Jin * \\ Jilin Key Laboratory of Solid-State Laser Technology and Application, School of Science, Changchun University \\ of Science and Technology, Changchun 130022, China; mayao@cust.edu.cn (Y.M.); xinchao@cust.edu.cn (C.X.); \\ zhangwei@cust.edu.cn (W.Z.) \\ * Correspondence: jgyciom@cust.edu.cn; Tel.: +86-431-85582465
}

\section{check for} updates

Citation: Ma, Y.; Xin, C.; Zhang, W.; Jin, G. Experimental Study of Plasma Plume Analysis of Long Pulse Laser Irradiates CFRP and GFRP

Composite Materials. Crystals 2021, 11, 545. https://doi.org/10.3390/ cryst11050545

Academic Editors: Anna

Paola Caricato, Renqin Dou,

Xiaoming Duan, Linjun Li and Xiaotao Yang

Received: 24 March 2021

Accepted: 2 May 2021

Published: 13 May 2021

Publisher's Note: MDPI stays neutra with regard to jurisdictional claims in published maps and institutional affiliations.

Copyright: (c) 2021 by the authors. Licensee MDPI, Basel, Switzerland. This article is an open access article distributed under the terms and conditions of the Creative Commons Attribution (CC BY) license (https:// creativecommons.org/licenses/by/ $4.0 /)$.

\begin{abstract}
The application of laser fabrication of fiber-reinforced polymer (FRP) has an irreplaceable advantage. However, the effect of the plasma generated in laser fabrication on the damage process is rarely mentioned. In order to further study the law and mechanism of laser processing, the laser process was measured. CFRP and GFRP materials were damaged by a $1064 \mathrm{~nm}$ millisecond pulsed laser. Moreover, the propagation velocity and breakdown time of plasma plume were compared. The results show that GFRP is more vulnerable to breakdown than CFRP under the same conditions. In addition, the variation of plasma plume and material surface temperature with the number of pulses was also studied. The results show that the variation trend is correlated, that is, the singularities occur at the second pulse. Based on the analysis of experimental phenomena, this paper provides guidance for plasma phenomena in laser processing of composite materials.
\end{abstract}

Keywords: long pulse laser; plasma plume; composite materials; CFRP; GFRP

\section{Introduction}

A composite material is usually defined as a combination of two or more different materials that have different physical and chemical properties. Thus, composite materials may possess the superior properties of both components [1,2]. Generally, in the fiberreinforced polymer (FRP), fiber wrapped by the resin matrix is the main load-bearing component of the material. At the same time, the resin is the load transfer element, which also plays the role of protecting the whole structure [3,4].

The properties of high specific strength and high modulus make fiber-reinforced resin composites useful in many fields, such as military, aviation, and sport equipment [5]. Because this kind of material is composed of fiber and matrix layering, there are several factors that influence the stiffness and strength of the composite material. For example, the direction of the fiber, the weaving method, the layering method, and the number of layers. Theoretically, there are many reinforcements and matrix materials that can be combined in a huge number of ways to produce composite structures that match specific applications of the structure. Fiber-reinforced lamination is composed of multilayer unidirectional or bidirectional fibers located within the matrix. Normally, materials should have similar properties (isotropy) in different directions, that is, they should have the same properties (homogeneity) at any point in the material. This means that the properties of the material are the same regardless of the direction and position of the material. However, when they are glued together and new special structures emerge, the properties are different [6]. Unidirectional fiber-reinforced materials exhibit different properties in the transverse direction of the fibers, while bidirectional materials exhibit different properties in all directions [7-12].

In the traditional middle infrared thermal imaging technology, the thermal imager records the mid-infrared radiation emitted by the object itself, which contains the characteristic information of the material; the difference of the mid-infrared radiation ability of 
material surfaces is caused by the change of the temperature field of the material surface. Usually, in the absence of external thermal excitation, the internal loss of the material will be reduced. Defects could not cause the temperature field change of the material surface, so the passive thermal imaging technology could not detect the defects under the material surface. Different from the traditional passive mid-infrared thermal imaging technology, the thermal wave detection technology uses the active control of light, heat, and other ways to stimulate the defects. The results show that the physical properties of different material surfaces and under the surface will affect the transmission of the heat wave and reflect the change of temperature field on the material surface in some way. By controlling the thermal excitation method and recording the infrared radiation of the material surface, the nonuniformity of the material can be obtained $[13,14]$.

In order to achieve the purpose of detection and flaw detection, the mechanism of laser induced damage of CFPR needs to be further explored. These processes of laser irradiation of fiber-reinforced polymers is very complicated, which is different from that of isotropic material or unistructural material. First, the material is heated by a laser, and the surface of the composite material is heated rapidly. Then, the surface of the resin reaches its decomposition temperature, its chemical bonds break, and decomposition occurs. Then, the material continues to absorb laser energy [15], resulting in combustion or gasification. Finally, the laser disintegrates and ionizes the combustion products to form plasma.

Therefore, in this paper, the experimental content proposed is to measure the temperature and combustion wave changes of FRP materials under laser damage in real time to obtain the process and law of long pulse lasers acting on FRP materials. In addition, the effects of different components, different structures, and different laser parameters on processing are compared, so as to provide technical reference for long pulse laser processing of FRP materials.

\section{Materials and Methods}

Melar 10, Nd: YAG, a millisecond pulse laser (Beamtech Optronics Co., Ltd, Beijing, China), was set up for the emitting laser. The laser wavelength was $1064 \mathrm{~nm}$, the pulse frequency was $10 \mathrm{~Hz}$, the pulse width was $0.5-3 \mathrm{~ms}$, and the energy was variable from $0.5-10 \mathrm{~J}$. The laser was partially reflected to the energy meter probe after passing the beam splitting mirror to measure the laser energy of the material in real time. At the same time, a KMGA740 high-speed thermometer was used to measure the temperature at the center point of the material surface. Meanwhile, the plasma generated in the experiment was photographed using the shadow method using the Phamton high speed camera (Vision Research, Inc., New York, NY, USA). The reference light was the $532 \mathrm{~nm}$ green laser, which propagated from the beam expanding mirror and the focusing lens. The frame frequency of the high-speed camera was 18,000 fps, and the exposure time was $10 \mathrm{~s}$ (see Figure 1).

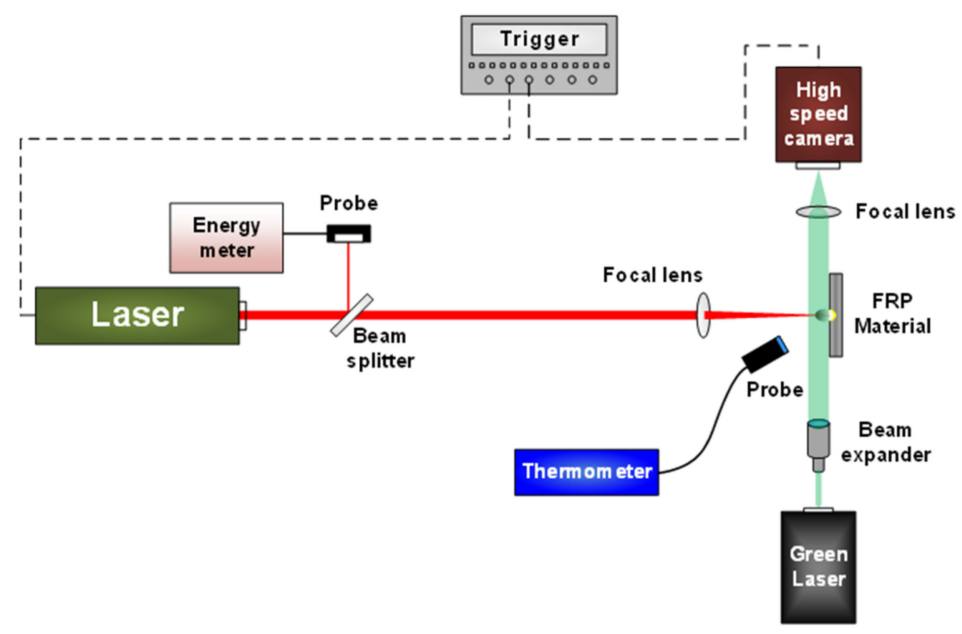

Figure 1. Experimental set-up. 
Carbon fiber-reinforced polymer (CFRP) and glass fiber-reinforced polymer (GFRP) were used in the experiment. The gasification temperatures of the two materials are $3500{ }^{\circ} \mathrm{C}$ and $1000{ }^{\circ} \mathrm{C}$, respectively. At the same time, the material structure is a multi-layer orthogonal bidirectional and unidirectional structure. The material is black sheet, which is composed of a thin layer of fiber and epoxy resin superimposed and solidified, with a surface size of $50 \mathrm{~mm} \times 50 \mathrm{~mm}$ and a thickness of $2 \mathrm{~mm}$.

\section{Results and Discussion}

\subsection{Plasma Plume Analysis}

When multiple laser pulses were applied to the composite surface, the material was broken down. A high-speed camera was used to take pictures of the laser action process to obtain the corresponding pulse number of the material breakdown (the frame frequency of the high-speed camera was $18,000 \mathrm{fps}$, and the exposure time was $10 \mathrm{~s}$ ) and to analyze the combustion wave morphology. At the same time, the propagation velocity of combustion waves on the front and back surfaces of the materials was measured to obtain the propagation law of combustion waves.

The shadow of ejected vapor does not appear on the back surface of the material, as shown in Figure 2a. However, As can be seen from the morphology, tense ejected material appears on both CFRP and GFRP surfaces, and this part of the material not only exists on the front surface but also appears on the back surface of the material, as shown in Figure 2b. This is because when the 14th pulse acted on CFRP, the plasma and spatter concentration on the back surface was low, which had dissipated after the end of the laser pulse, so no significant shadow was formed.

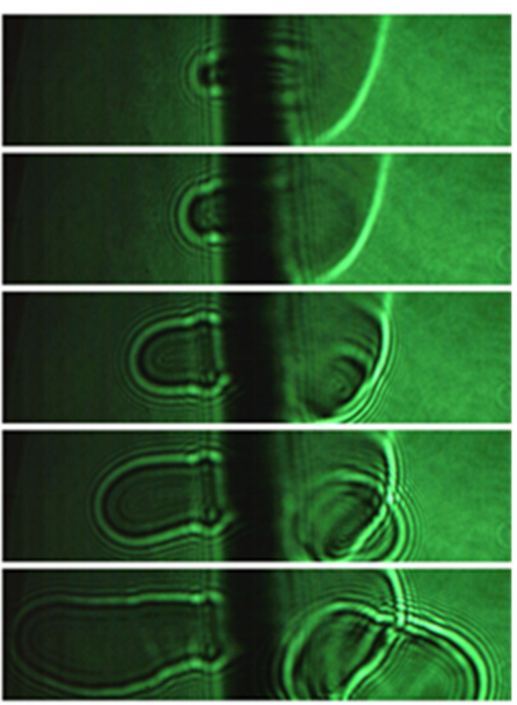

(a)

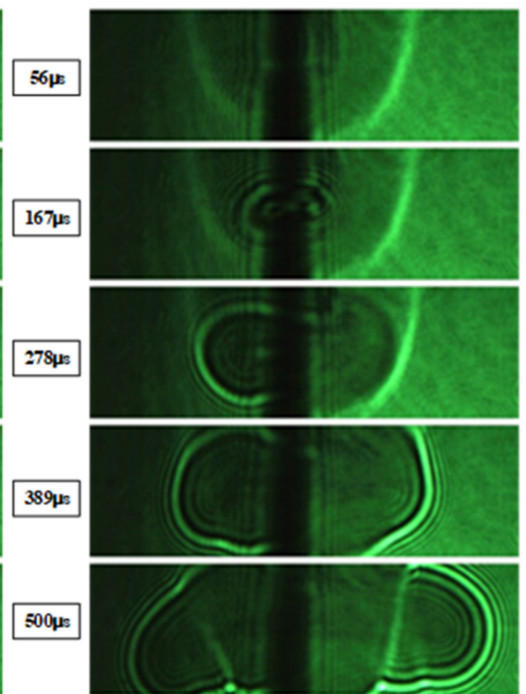

(b)

Figure 2. Combustion wave morphology (a) unidirectional CFRP, $\tau \mathrm{p}=1 \mathrm{~ms}, \mathrm{~N}=20, \mathrm{n}=14$ th, $\mathrm{d}=2 \mathrm{~mm}, \mathrm{~F}=392 \mathrm{~J} / \mathrm{cm}^{2}$. (b) Unidirectional GFRP, $\tau \mathrm{p}=1 \mathrm{~ms}, \mathrm{~N}=20, \mathrm{n}=13 \mathrm{th}, \mathrm{d}=2 \mathrm{~mm}$, $\mathrm{F}=392 \mathrm{~J} / \mathrm{cm}^{2}$.

At the same time, it can be seen in Figure 2a that when the laser irradiated on the material surface with ejected vapor, the plasma expands slowly. When the combustion wave keeps moving outside the ejected vapor, its motion direction changes. This is because the interior structure of the ejected material is complex and uneven. When the laser penetrates the surface of the material, it is no longer the original waveform. At the same time, the process of outward expansion is also affected by the internal components of the ejected material, so the angle is offset.

Figure $3 a, b$ show the combustion wave expansion velocity of CFRP materials during 20 pulses. Under the same laser conditions, the bidirectional material is penetrated at 
the 12th pulse and unidirectional at the 10th pulse. In the process of laser irradiating, the expansion velocity on the back surface is slow in the initial period and increases in the later period. In the early period of material penetration, only few laser energies can penetrate the plasma inside the keyhole and act on the back surface, thus producing insufficient energy. When the backside material is badly ablated, more laser energy can penetrate the material and act on the plasma on the back surface, so the combustion wave velocity is faster. This process is similar to the situation on the front surface in which the speed first increases and then decreases. This phenomenon conforms to the law of energy conservation. At the same time, by comparing the two different structures, the material of the bidirectional structure was broken down in the 12th pulse, while the unidirectional material was broken down in the 10th pulse. From Figure 3c,d, due to the number of pulses, the bidirectional structure is less likely to be damaged for the CFRP material.
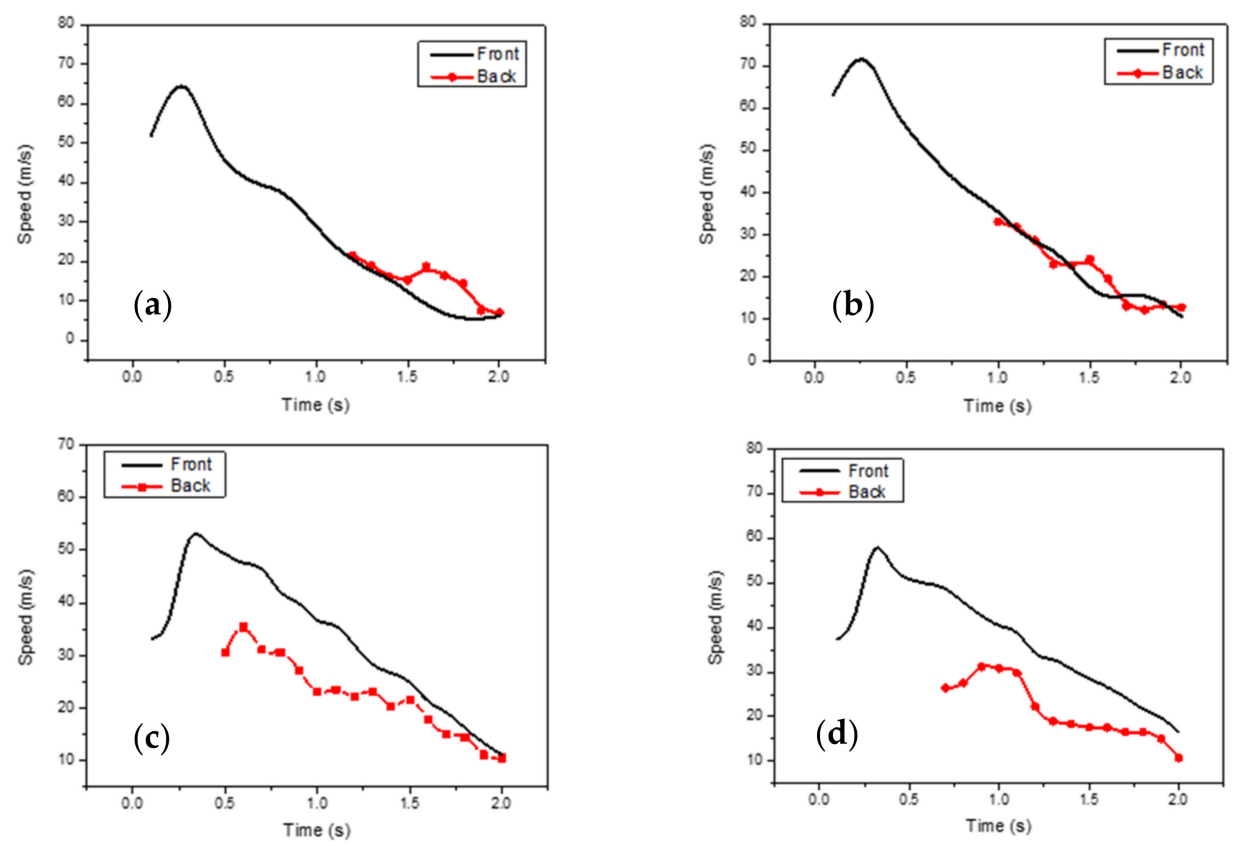

Figure 3. Combustion wave expansion velocity changing with time ( $\tau \mathrm{p}=1 \mathrm{~ms}, \mathrm{~N}=20, \mathrm{~d}=2 \mathrm{~mm}$, $\mathrm{F}=392 \mathrm{~J} / \mathrm{cm}^{2}$ ). (a) Bidirectional CFRP; (b) Unidirectional CFRP; (c) Bidirectional GFRP; (d) Unidirectional GFRP.

Figure $3 c$,d show the combustion wave expansion velocity of GFRP materials during 20 pulses. By comparing that to the CFRP materials, the trend is similar. However, bidirectional material is broken at the 5th pulse, while the unidirectional GFRP is broken at the 7th pulse. Under the same laser conditions, the bidirectional structure GFRP material is more easily broken down.

Figure 4 shows the relationship of combustion wave expansion velocity changing with time of FRP materials, and the dots in the figure show the breakdown time of the material. By comparing the action law before breakdown, the plasma expansion velocity of CFRP material decreases with the increase in the number of pulses and only increases when the second or third pulse occurs. However, GFRP material increases with the number of pulses. By comparing the breakdown time of the two materials, GFRP material was broken before CFRP material, regardless of the material structure. In addition, the comparison of the expansion velocity shows that the velocity of CFRP in the early stage is greater than that of GFRP, and that of GFRP in the late stage is greater than that of CFRP. 

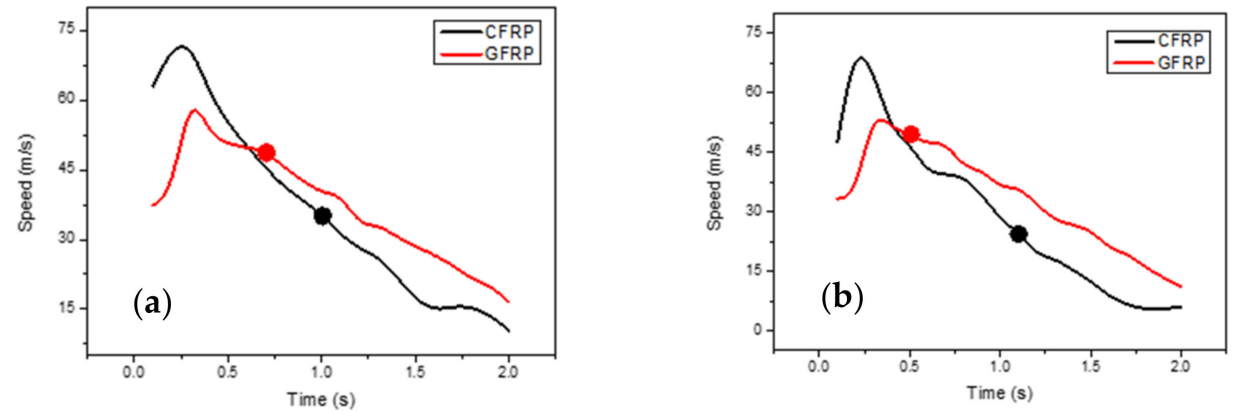

Figure 4. Combustion wave expansion velocity changing with time ( $\tau \mathrm{p}=1 \mathrm{~ms}, \mathrm{~N}=20, \mathrm{~d}=2 \mathrm{~mm}$, $\mathrm{F}=392 \mathrm{~J} / \mathrm{cm}^{2}$ ), (a) Unidirectional; (b) Bidirectional.

\subsection{Temperature Analysis}

In the experiment, the surface area of material was measured. Under the action of the multi-pulse laser, the material is injected with laser energy, so that the temperature of the material rises until decomposition, gasification, and even ionization. When the pulse laser fluence is $392 \mathrm{~J} / \mathrm{cm}^{2}$, a single pulse can gasify and ionize the FRP material, resulting in an ablative zone. Therefore, plasma is sensitive to laser conditions; because of the generation time of the plasma difference in the two materials the plasma is sometimes beneficial to heat conduction, but sometimes it can be the opposite due to the plasma shielding effect.

In laser processing of composite materials, a multi-pulse laser is often used to irradiate them to improve the processing efficiency by utilizing the accumulation effect of temperature and plasma. Therefore, in the research of multi-pulse laser processing, the residual temperature of the material surface after each pulse ends while the next pulse arrives is considered to represent the temperature accumulation effect of the material, so as to analyze the temperature during the processing of the material.

Figure 5 shows the curve of the residual temperature of CFRP and GFRP during 20 laser pulses. The overall trend is that the residual temperature increases with the number of pulses, and the accumulative trend is obvious. However, at the same laser fluence, the residual temperature of GFRP material is higher than that of CFRP, and the temperature of GFRP decreases in the second pulse, while that of CFRP continues to rise.

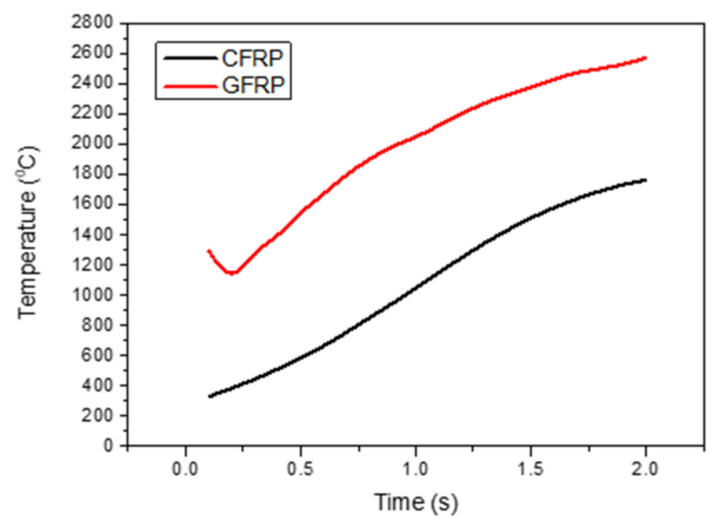

Figure 5. Residual temperature changing with time when laser damaged FRP materials $(\tau p=1 \mathrm{ms,}$ $\left.\mathrm{N}=20, \mathrm{~d}=2 \mathrm{~mm}, \mathrm{~F}=392 \mathrm{~J} / \mathrm{cm}^{2}\right)$.

From the figure, the residual temperature of the two materials is quite different. The CFRP materials fall in temperature below the gasification temperature $\left(3500{ }^{\circ} \mathrm{C}\right)$, which is higher than the plasma temperature $\left(104^{\circ} \mathrm{C}\right)$. This shows that when the next pulse arrives, plasma dissipated toward an inverse direction of laser propagation. Thus, the plasma density is low, which cannot form the plasma shielding effect of the laser. On the contrary, the residual temperature of GFRP material is much higher than that of the material gasification point $\left(1000^{\circ} \mathrm{C}\right)$, so the measured temperature should be a mixture of material 
decomposition and plasma. This is because when compared to carbon fiber, glass fiber is mainly composed of $\mathrm{SiO}_{2}$, which is usually amorphous and has a higher melting point and damage threshold. Thus, GFRPs are more likely to produce a higher concentration of the mixture than CFRPs, while the mixture shields the incident laser. After that, laser energy irradiated on the composite material surface decreases, and the cumulative effect becomes weak in a short period of time. However, when the subsequent pulsed laser continues to irradiate, the mixture in front of the material surface is ignited by the laser, and the combustion wave propagates in the opposite direction, which weakens the shielding effect. Thus, the laser energy can re-pass through the mixture and act on the surface of the material, thus intensifying the effect and promoting the temperature accumulation.

\subsection{Correlation Analysis}

Based on the analysis of plasma plume and temperature, these two dependent variables both appear as singularities with the increase in the number of pulses. However, the difference is that for CFRP, the residual temperature curve is relatively smooth and there is no singularity. This is because the damage mechanism of the two materials is different when they are damaged by laser. Comparing the material properties of carbon fiber and glass fiber, we can see that the damage mechanism of carbon fiber and glass fiber is quite different.

The epoxy resin attached to the surface of the fiber has a higher laser transmittance than the fiber, so the laser directly acts on the fiber through the epoxy resin on the surface of the material [8]. Carbon fiber is an opaque medium, while glass fiber is a translucent medium. This difference results in the different transmittance of the material to the laser, that is, the transmittance of glass fiber is much higher than that of carbon fiber.

The electronic drift in CFPR and GFPR is disordered. The main reason for the difference between the two curves is lattice-lattice coupling. It is well known that the lattice of carbon in CFPR is periodic, while that of $\mathrm{SiO}_{2}$ in GFPR is aperiodic. Therefore, the thermal conductivity of the two materials is different under the same laser conditions.

Comparing the two kinds of fibers, the thermal conductivity of carbon fiber is $60 \mathrm{~W} / \mathrm{m} \cdot \mathrm{K}$, while that of glass fiber is only $1.09 \mathrm{~W} / \mathrm{m} \cdot \mathrm{K}$. According to the above two parameters, the speed of CFRP rising to the gasification point is much higher than that of GFRP during the heating process of laser activated material. However, the gasification point of the two is different. As we discussed above, glass fiber is mainly composed of $\mathrm{SiO}_{2}$, which is usually amorphous, it has no fixed melting point; when the same energy laser acts on the surface of the material and causes the material to heat up, the glass fiber is more likely to heat up to its gasification temperature and ionize. Glass fiber or other forms tend to form stable melts within a large range, and also change their viscosity gradually before they reach a liquid state. Carbon fiber tends to oxidize in air and pyrolysis in Ar atmospheres. In fact, the absorption of pure glass at $1064 \mathrm{~nm}$ is relatively low $[16,17]$. The plasma temperature produced by ionization is much higher than that of the surface temperature of the material. Meanwhile, based on the thermal conductivity factor, the cooling rate of CFRP materials is also faster. Therefore, at the end of each pulse, the residual temperature of CFRP is much lower than GFRP.

The plasma and temperature changes of the two materials were compared. There are singularity effects on temperature and plasma plume of GFRP materials. At the second pulse, the plume has a higher velocity and a lower temperature. Both defy the overall trend. When the laser acts on the material, the fiber acts as the main medium for heat exchange. Its temperature is first raised, and then the heat is transferred to the surrounding epoxy resin through heat conduction. Therefore, at the first pulse, although the temperature of the fiber first rises, it does not reach the gasification point, but the surrounding epoxy resin has decomposed and ionized. Under the continuous action of the laser, the fiber is vaporized and decomposed along with the epoxy, resulting in a flocculent mixture that floats on the surface of the material, which is dense and difficult to disperse. When the second pulse acts on the material, the mixture on the surface first receives the laser energy for secondary 
decomposition and ionization, which accelerates the propagation speed of the plasma plume. At the same time, due to the shielding of this part of the mixture, the material table can receive less laser energy, so there is an obvious cooling phenomenon. After that, when the subsequent pulses arrive, the shield on the surface of the material is thin because the previously thick mixture has largely decomposed and ionized. While there is still a case for absorbing subsequent lasers, most laser pulses can penetrate the shield and hit the material. Therefore, when the laser distribution of the two parts reaches a relatively balanced state, the subsequent variation trend of plasma plume and temperature tends to be monotonous.

\section{Conclusions}

In this paper, the temperature and combustion wave changes in FRP materials processed by a long pulse infrared laser are studied.

Under the multi-pulse conditions, the residual temperature of the material increased with the increase in the number of pulses for the two materials. In addition, the morphology of the plasma combustion wave was also analyzed when the material was broken down, and the phenomenon of the ejected vapor shadow and the deviation of the combustion wave propagation direction was found. However, the residual temperature of GFRP material was higher than that of CFRP under the same laser fluence. Moreover, the residual temperature of CFRP continued to rise, but the temperature of GFRP dropped in the second pulse due to the shielding effect. Finally, under the same laser conditions, CFRP material of a bidirectional structure was less likely to be damaged by the pulse train laser, while GFRP material of a bidirectional structure was more likely to be broken down. Due to the different structure of the materials, when the multi-pulse laser irradiates the surface of the carbon fiber material, the glass fiber material was decomposed before the carbon fiber material. As a result, when the laser parameters are precisely controlled, multi-pulse laser can process FRP materials more effectively due to the temperature accumulation effect and the formation of plasma.

Author Contributions: Conceptualization, Y.M. and C.X.; methodology, W.Z.; software, W.Z.; validation, Y.M., C.X., and W.Z.; formal analysis, Y.M.; investigation, Y.M.; resources, Y.M.; data curation, Y.M.; writing_original draft preparation, G.J.; writing—review and editing, Y.M.; visualization, Y.M.; supervision, Y.M.; project ad funding acquisition, G.J. All authors have read and agreed to the published version of the manuscript.

Funding: This research was funded by National Natural Science Foundation of China, grant number U19A2077 and the Science and Technology Research project of the Jilin Provincial Department of Education (Grant No. JJKH20200732KJ).

Data Availability Statement: The data presented in this study are available on request from the corresponding author.

Acknowledgments: We thank the Jilin Key Laboratory of Solid-State Laser Technology and Application, School of Science, Changchun University of Science and Technology.

Conflicts of Interest: The authors declare no conflict of interest.

\section{References}

1. Rahman, M.; Ramakrishna, S.; Prakash, J.R.S.; Tan, D. Machinability study of carbon fiber reinforced composite. J. Mater. Process. Technol. 1999, 89, 292-297. [CrossRef]

2. William, F.; Smith Hashemi, J.; Presuel-Moreno, F. Foundations of Materials Science and Engineering; Mcgraw-Hill Publishing: New York, NY, USA, 2006.

3. King, R.L. Fibre-Reinforced Composites Materials, Manufacturing and Design. Composites 1989, 20, 172-173. [CrossRef]

4. Mitchell, B.S. An Introduction to Materials Engineering and Science for Chemical and Materials Engineers; John Wiley \& Sons: Hoboken, NY, USA, 2004.

5. Karataş, M.A.; Gökkaya, H. A review on machinability of carbon fiber reinforced polymer (CFRP) and glass fiber reinforced polymer (GFRP) composite materials. Def. Technol. 2018, 14, 318-326. [CrossRef]

6. Yung, K.C.; Mei, S.M.; Yue, T.M. A study of the heat-affected zone in the UV YAG laser drilling of GFRP materials. J. Mater. Process. Technol. 2002, 122, 278-285. [CrossRef] 
7. Ohkubo, T.; Sato, Y.; Matsunaga, E.; Tsukamoto, M. Three-dimensional numerical simulation during laser processing of CFRP. Appl. Surf. Sci. 2017, 417, 104-107. [CrossRef]

8. Takahashi, K.; Tsukamoto, M.; Masuno, S.; Sato, Y. Heat conduction analysis of laser CFRP processing with IR and UV laser light. Compos. Part A Appl. Sci. Manuf. 2016, 84, 114-122. [CrossRef]

9. Abrao, A.M.; Rubio, J.C.; Faria, P.E.; Davim, J.P. The effect of cutting tool geometry on thrust force and delamination when drilling glass fibre reinforced plastic composite. Mater. Des. 2008, 29, 508-513. [CrossRef]

10. Tsao, C.C.; Hocheng, H. Effect of tool wear on delamination in drilling composite materials. Int. J. Mech. Sci. 2007, 49, 983-988. [CrossRef]

11. Durão, L.M.P.; Gonçalves, D.J.; Tavares, J.M.R.; de Albuquerque, V.H.C.; Vieira, A.A.; Marques, A.T. Drilling tool geometry evaluation for reinforced composite laminates. Compos. Struct. 2010, 92, 1545-1550. [CrossRef]

12. Goeke, A.; Emmelmann, C. Influence of laser cutting parameters on CFRP part quality. Phys. Procedia 2010, 5, 253-258. [CrossRef]

13. Abrão, A.M.; Faria, P.E.; Rubio, J.C.; Reis, P.; Davim, J.P. Drilling of fiber reinforced plastics: A review. J. Mater. Process. Technol. 2007, 186, 1-7. [CrossRef]

14. Zhukova, E.S.; Zhang, H.; Martovitskiy, V.P.; Selivanov, Y.G.; Gorshunov, B.P.; Dressel, M. Infrared Optical Conductivity of Bulk $\mathrm{Bi}_{2} \mathrm{Te}_{2}$ Se. Crystals 2020, 10, 553. [CrossRef]

15. Liu, J.-X.; Mei, S.-L.; Chen, X.-H.; Yao, C.-J. Recent Advances of Near-Infrared (NIR) Emissive Metal Complexes Bridged by Ligands with N- and/or O-Donor Sites. Crystals 2021, 11, 155. [CrossRef]

16. Huang, Z.Q.; Hong, M.H.; Do, T.B.M.; Lin, Q.Y. Laser etching of glass substrates by 1064 nm laser irradiation. Appl. Phys. A 2008, 93, 159-163. [CrossRef]

17. Xu, H.B.; Hu, J.; Yu, Z. Absorption behavior analysis of Carbon Fiber Reinforced Polymer in laser processing. Opt. Mater. Express. 2015, 5, 2330-2336. [CrossRef] 